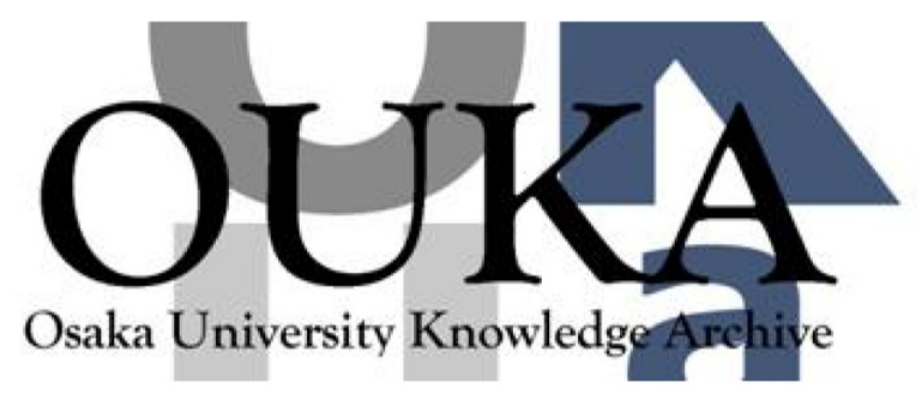

\begin{tabular}{|c|l|}
\hline Title & $\begin{array}{l}\text { Visual-area coding technique (VACT) : optical } \\
\text { parallel implementation of fuzzy logic and its } \\
\text { visualization with the digital-halftoning } \\
\text { process }\end{array}$ \\
\hline Author(s) & $\begin{array}{l}\text { Konishi, Tsuyoshi; Tanida, Jun; Ichioka, } \\
\text { Yoshiki }\end{array}$ \\
\hline Citation & Applied Optics. 34(17) p. 3097-p. 3102 \\
\hline Issue Date & 1995-06-10 \\
\hline oaire:version & VoR \\
\hline URL & https://hdl.handle. net/11094/3100 \\
\hline rights & \\
\hline Note & \\
\hline
\end{tabular}

Osaka University Knowledge Archive : OUKA

https://ir. Library. osaka-u. ac. jp/

Osaka University 


\title{
Visual-area coding technique (VACT): optical parallel implementation of fuzzy logic and its visualization with the digital-halftoning process
}

Tsuyoshi Konishi, Jun Tanida, and Yoshiki Ichioka

\begin{abstract}
A novel technique, the visual-area coding technique (VACT), for the optical implementation of fuzzy logic with the capability of visualization of the results is presented. This technique is based on the microfont method and is considered to be an instance of digitized anal og optical computing. Huge amounts of data can be processed in fuzzy logic with the VACT. In addition, real-time visualization of the processed result can be accomplished.

Key words: Optical computing, digitized analog computing, fuzzy logic, digital halftoning, visualization.
\end{abstract}

\section{Introduction}

Optics has excellent features such as inherent parallelism, high processing speeds, three-dimensional interconnection capabilities, and broad bandwidths for information transmission and processing. Through good use of these features of optics, digital optical computing has become an increasingly promising research field. ${ }^{1-8}$ Optical implementation of logical operation is an important subject in digital optical computing. For example, optical-array logic, ${ }^{1}$ symbolic substitution, ${ }^{2}$ bi nary-image algebra, ${ }^{3}$ and imageIogic algebra 4 aretypical techniques for the implementation of optical parallel logic.

Digital optical systems have excellent features, such as high accuracy, flexibility, and programmability, when compared with analog optical systems. On the other hand, analog-computing techniques, i.e., neuro processing, fuzzy processing, chaos, and fractal processing, have become increasingly attractive applications. To use both processing schemes, we consider a novel concept of processing, digitized analog computing. A digitized analog optical-computing system is defined as a digital optical system specified for analog data processing. Several techniques and methods have been presented on this

The authors are with the Department of Applied Physics, Faculty of Engineering, Osaka University, 2-1 Yamadaoka, Suita, Osaka 565, J apan.

Received 25 August 1994; revised manuscript received 22 November 1994.

0003-6935/95/173097-06\$06.00/0.

(c) 1995 Optical Society of America. concept. ${ }^{9,10}$ Recently, optical digital implementation of fuzzy logic that is based on the area coding technique $(A C T)$ has been proposed. ${ }^{11}$ The ACT is a typical example of digitized analog optical computing.

Now let us consider a potential feature of optical computing. As is well known, optical parallel-logic gates treat image data as an information medium for parallel processing. An image has an inherent capacity to provide a visual interface for humans. Therefore, it is expected that an effective visual-interface system can be implemented by optical parallel computing. In other words, the availability of visual techniques provides new applications for optical computing in which data visualization is essential.

In this paper, we propose a novel coding technique, the visual-area coding technique (VACT), for a digitized analog optical computing system; it enables us to visualize the results of processing. Section 2 presents the ACT and the microfont method (MFM) on which the VACT is based. Section 3 presents principles of the VACT. Experimental result are de scribed in Section 4 and demonstrate that fuzzy reasoning is an instance of the applications of the VACT.

\section{Area-Coding Technique and Microfont Method}

In the ACT, the gray value is represented as the area of the transparent part in a pixel, as shown in Fig. 1.11 Processing in the ACT is based on fuzzy logic. Fuzzy logic is an extension of set-theoretic multivalued logic in which the truth values are presented by linguistic variables. $^{12}$ The fundamental operations in fuzzy logic are the maximum, minimum, and negation 


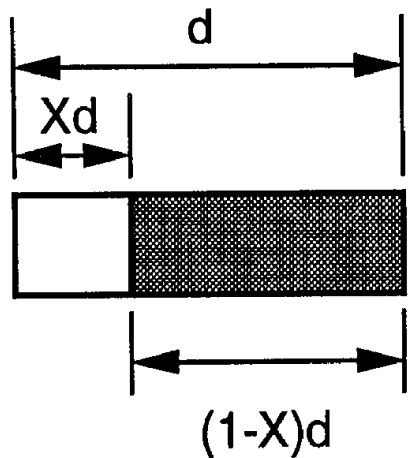

Fig. 1. Representation of the gray value $X$ with theACT.

operations for fuzzy sets, given by

$$
\begin{aligned}
\mu(A) \vee \mu(B) & =\max [\mu(A), \mu(B)], \\
\mu(A) \wedge \mu(B) & =\min [\mu(A), \mu(B), \\
-\mu(A) & =1-\mu(A),
\end{aligned}
$$

where $\mu(A)$ and $\mu(B)$ are fuzzy membership functions representing fuzzy sets and $\vee, \wedge$, and $\neg$ denote the mean maximum, minimum, and negation operators, respectively. $\mu(X)$ is a function whose value is restricted within the closed interval $[0,1]$.

From the point of view of the coding methodology, the code pattern used in the ACT is a kind of direct representation of numerical information. In theACT, the gray value is represented by the aggregated bright area. In contrast, distributed representation of the numerical information can be considered, and it is mainly used in the digital-halftoning process. Digital halftoning is a practical method that renders an illusion of continuous-tone images on binary display devices. ${ }^{13}$ Concrete methods of the digital-halftoning process, e.g., pulse-amplitude modulation, pulse-surface-area modulation, ordered dither, and MFM, have been proposed. ${ }^{13-15}$ Among these, the MFM is suitable to render detailed pictures. The MFM is a technique that renders the gray value by conversion of the value into a specific microfont. In the MFM three typical sets of fonts are used: Bayer, Spiral, and Net. The Bayer font set for 17 gray tones is shown in Fig. 2.

Figure 3 shows the rendering process with the Bayer font set. First, each gray value of an input pixel is assigned a threshold value by a threshold-

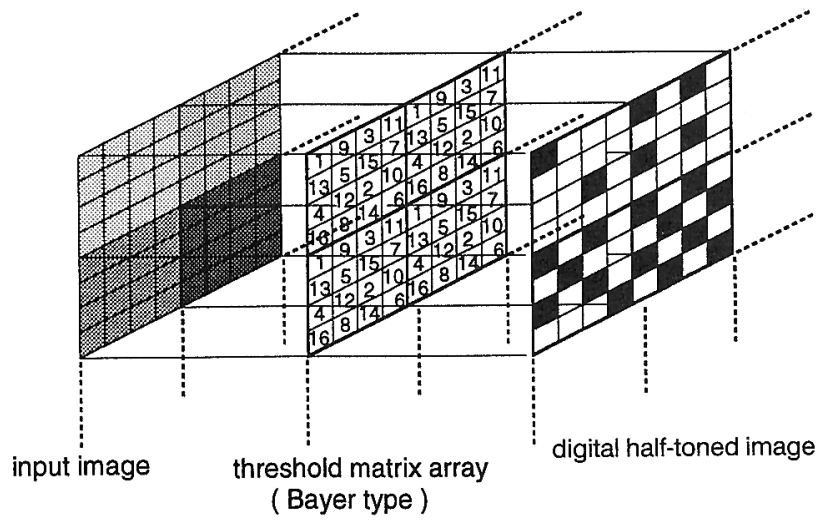

Fig. 3. Rendering process of the MFM.

matrix array whose elements are determined by the Bayer font set. After passing through the thresholdmatrix array, each gray value is converted into a set of binary patterns corresponding to the gray value. Note that both the ACT and digital halftoning are considered to be good examples of digitized analog processing. Therefore, it is expected that a new digitized analog optical-computing technique can be developed through their concepts.

\section{Visual-Area Coding Technique}

The novel VACT for digitized analog optical computing provides parallel maximum, minimum, and negation operations whose results can be visually observed at any time. Figure 4 shows the processing procedure for optical parallel implementation of the maximum, minimum, and negation operations with the VACT. First, in the halftoning process, a given discrete continuous-tone image is transformed into a halftoned image. An individual pixel datum is coded with a threshold-matrix array. A threshold-matrix array corresponds to the font set of the MFM. In the VACT, the Bayer font set is used. Second, in the logic-conversion process, the contrast of the halftoned image is inverted for the case of minimum operation. Third, in the discrete-correlation process, a discrete correlation is executed between the halftoned image and kernel-pattern operation. This procedure corresponds to the shadow-casting step in the ACT.11 The result of the maximum operation is obtained directly after discrete correlation, whereas additional inversion is required for the minimum operation.

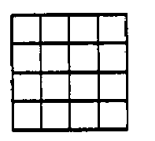

0

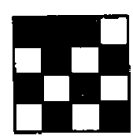

9

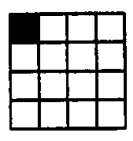

1

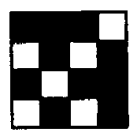

10
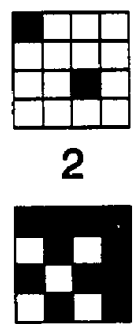

11

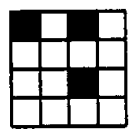

3

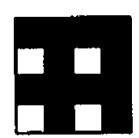

12
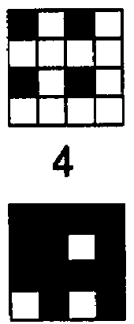

13

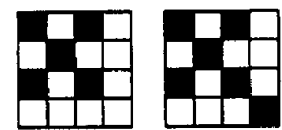

5

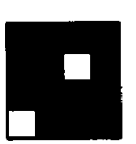

14

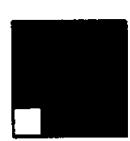

15

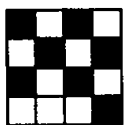

7

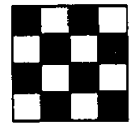

8

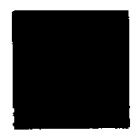

16

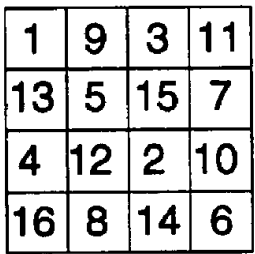

threshold matrix array (Bayer type)

Fig. 2. Bayer font set of the MFM with 17 gray tones. 


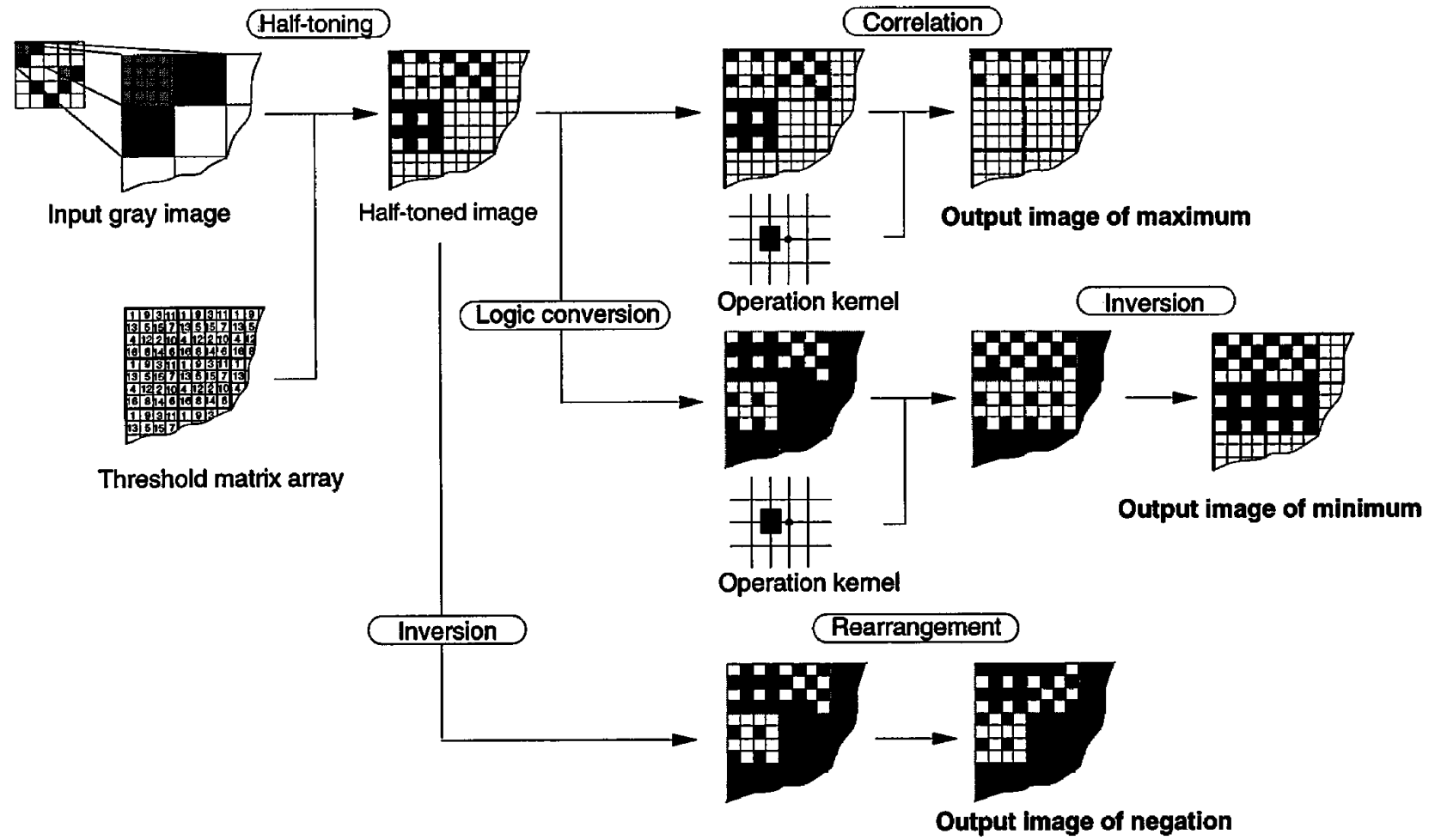

Fig. 4. Processing procedure for optical parallel implementation of fuzzy logic with the VACT.

In the ACT, the negation operation is achived by contrast inversion of a coded pattern. In the VACT, however, positional inversion is also required. The negation operation in the VACT must not only invert the contrast in a coded pattern but al so rearrange the distribution within a coded pattern. This rearrangement of the distribution is implemented when the distribution is turned upside down, as given by

$$
P_{k}, I=P_{k},(m-I+1), \quad 1 \leqq k \leqq m, \quad 1 \leqq I \leqq m,
$$

where $P_{k}, I$ is the value of the $(k, I)$ th subpixel within a coded pattern. Figure 5 shows the processing procedure of the negation operation for the case of $\mathrm{m}=4$. Figure 6 shows an optical setup for parallel implementation of the negation operation with the VACT. Contrast inversion of a coded pattern is implemented with a cross-Nicol arrangement of a spatial-light modulator and a polarizing beam splitter. The rearrangement of the distribution is implemented with a patterned mirror. ${ }^{16}$ The pitch of patterned mirror is set to be equal to the size of pixel.

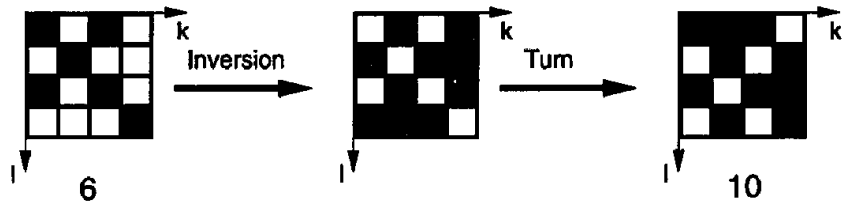

Fig. 5. Processing procedure of the negation operation with the VACT.
To digitize the continuous value in the VACT, the closed interval $[0,1]$ must be quantized by the number of gray tones. For the case of 17 gray tones, for example, the width of the discrete level corresponds to 0.0625 . This value is equal to one sixteenth of the closed interval.

Fuzzy processing that is based on fuzzy logic or on gray-scale image processing with mathematical morphology can be considered to be an application of the VACT. 11,17,18 Fuzzy reasoning is an effective application. In the design and tuning processes of a fuzzy control system that is based on fuzzy reasoning, visualization of the result of fuzzy reasoning is ex-

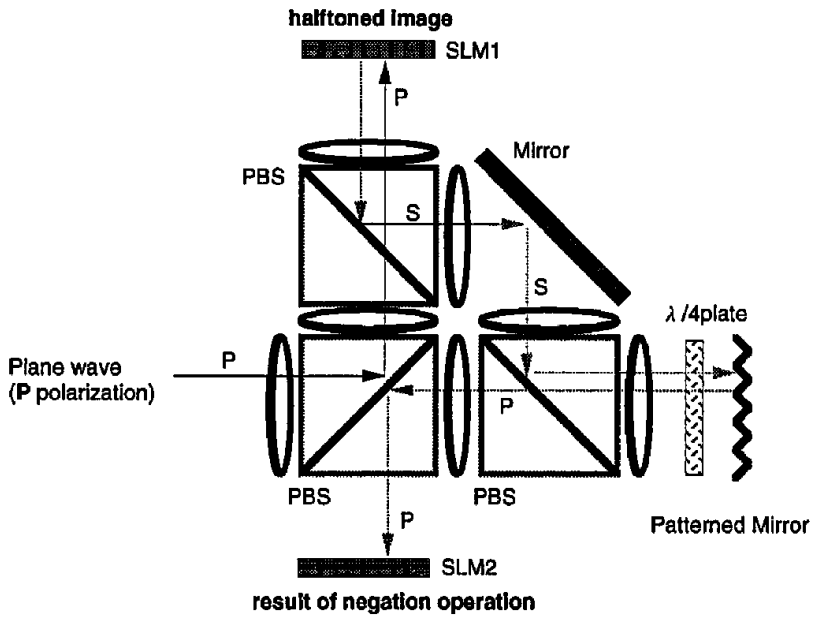

Fig. 6. Optical setup for parallel implementation of the negation operation.

10 June 1995 / Vol. 34, No. 17 / APPLIED OPTICS

3099 
$\mu(A): A=\left[\begin{array}{l}0.00 \\ 0.50 \\ 0.68 \\ 1.00 \\ 0.88 \\ 0.75 \\ 0.63 \\ 0.44 \\ 0.25 \\ 0.00\end{array}\right] \quad W=$

(a)

$\mu(B): B=\left[\begin{array}{l}0.00 \\ 0.13 \\ 0.31 \\ 0.50 \\ 0.75 \\ 0.88 \\ 1.00 \\ 0.63 \\ 0.38 \\ 0.00\end{array}\right]$

(b)

$\mu\left(A^{\prime}\right): A^{\prime}=\left[\begin{array}{c}0.00 \\ 0.38 \\ 0.63 \\ 1.00 \\ 0.88 \\ 0.75 \\ 0.50 \\ 0.31 \\ 0.13 \\ 0.00\end{array}\right]$

(c)
$0.00,0.00,0.00,0.00,0.00,0.00,0.00,0.00,0.00,0.00$

$0.00,0.13,0.13,0.13,0.13,0.13,0.13,0.13,0.13,0.00$

$0.00,0.31,0.31,0.31,0.31,0.31,0.31,0.31,0.25,0.00$

$0.00,0.50,0.50,0.50,0.50,0.50,0.50,0.44,0.25,0.00$

$0.00,0.50,0.69,0.75,0.75,0.75,0.63,0.44,0.25,0.00$

$0.00,0.50,0.69,0.88,0.88,0.75,0.63,0.44,0.25,0.00$

$0.00,0.50,0.69,1.00,0.88,0.75,0.63,0.44,0.25,0.00$

$0.00,0.50,0.63,0.63,0.63,0.63,0.63,0.44,0.25,0.00$

$0.00,0.38,0.38,0.38,0.38,0.38,0.38,0.38,0.25,0.00$

$0.00,0.00,0.00,0.00,0.00,0.00,0.00,0.00,0.00,0.00$

(d)

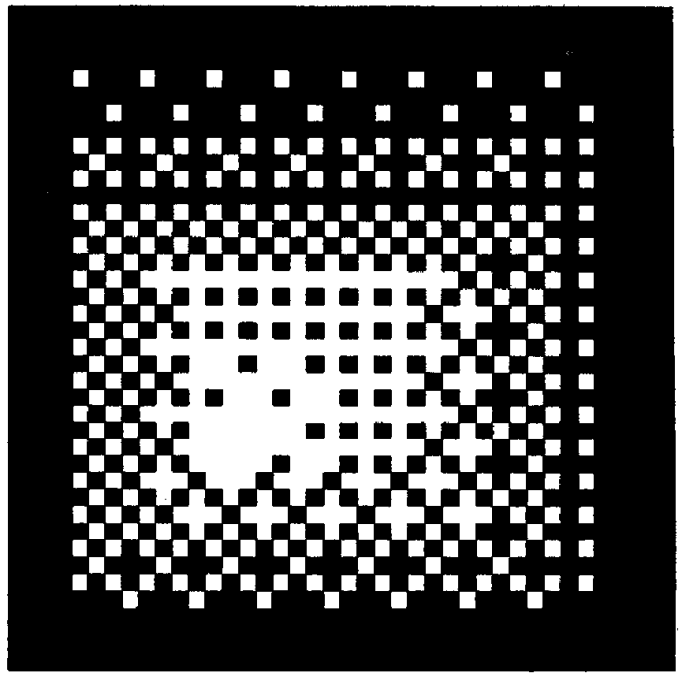

(e)
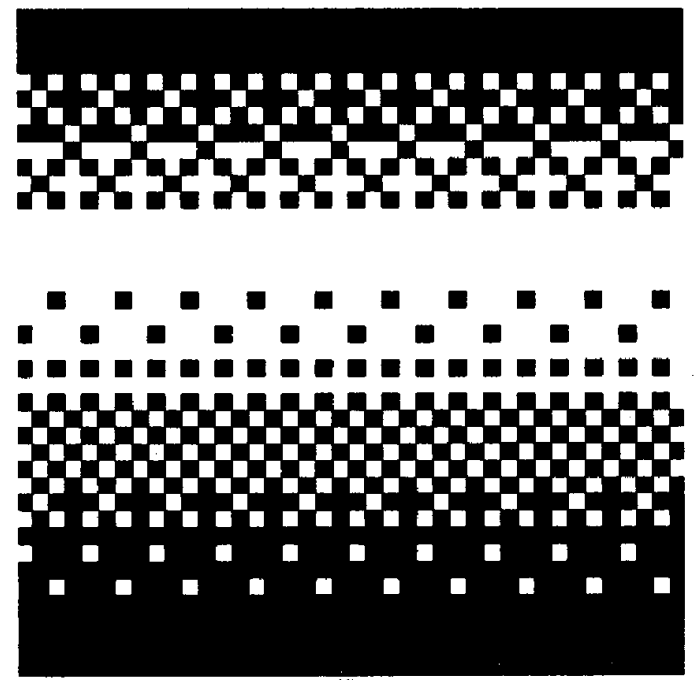

(f)

Fig. 7. Experimental elements for fuzzy sets A and B: (a) vector A, (b) vector B, (c) fuzzy relation matrix $\mathbf{W}$, (d) coded pattern of $\mathbf{W},(\mathrm{e})$ input vector $\mathbf{A}^{\prime}$, and $(\mathbf{f})$ expansion of the coded pattern of $\mathbf{A}^{\prime}$. 


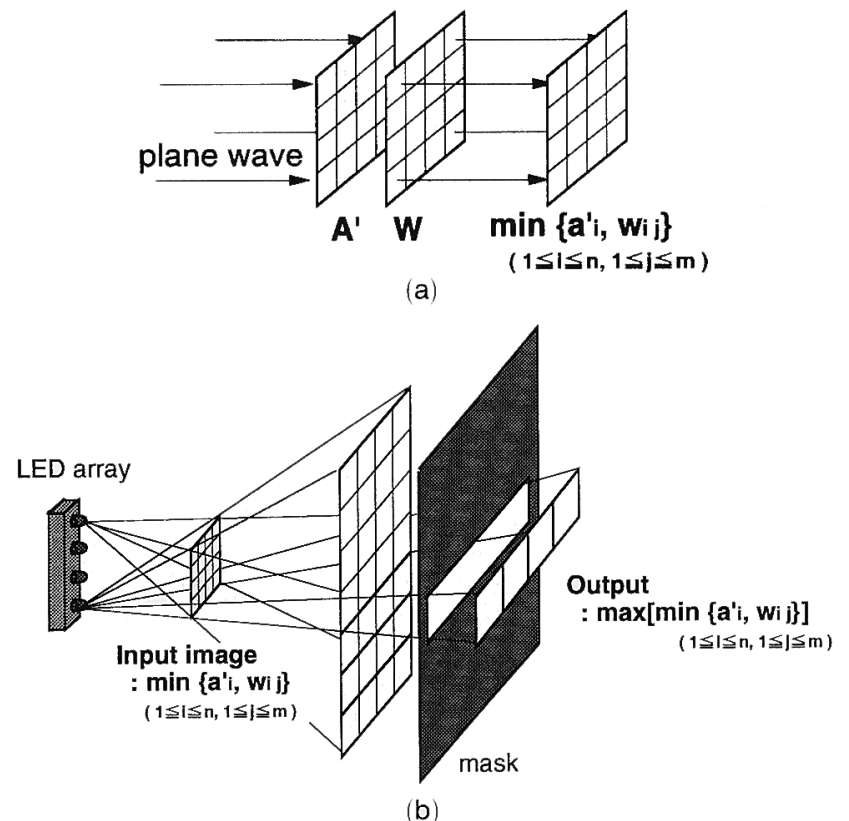

Fig. 8. Experimental setup for the $(a) \min \left\{a_{\mathrm{i}}^{\prime}, w_{i j}\right\}$ operation, and $(b)$ $\max \left[\min \left[a_{i}^{\prime}, w_{i j}\right]\right]$ operation.

pected to be useful to monitor complex system dynamics. ${ }^{19,20}$

\section{Experimental Results}

To verify the principle of the VACT, we executed an optical experiment on fuzzy reasoning. Fuzzy reasoning is a processing operation to reason a subject from a fuzzy rule between fuzzy sets $A$ and $B$. Fuzzy sets $A$ and $B$ are represented by vectors $\mathbf{A}=\left\{a_{1}, \ldots, a_{n}\right\}$ and $\mathbf{B}=\left\{b_{1}, \ldots, b_{m}\right\}$, where $a_{i}$ and $b_{j}$ are the values of the ith members of fuzzy set $A$ and the jth member of fuzzy set $B$, respectively. A fuzzy rule between fuzzy sets $A$ and $B$ is represented by a fuzzy relation, $W$. Fuzzy relation $\mathrm{W}$ is represented by the Cartesian product of fuzzy sets $\mathbf{W}$. The Cartesian product of fuzzy sets $W$ is a matrix in the product space $\mathbf{A} \times \mathbf{B}$. $\mathbf{W}$ and its element $\mathbf{w}_{\mathrm{ij}}$ are expressed as

$$
\begin{aligned}
\mathbf{W} & =\mathbf{A} \times \mathbf{B}, \\
\mathrm{w}_{\mathrm{ij}} & =\min \left[\mathrm{a}_{\mathrm{i}}, \mathrm{b}_{\mathrm{j}}\right], \quad 1 \leqq \mathrm{i} \leqq \mathrm{n}, \quad 1 \leqq \mathrm{j} \leqq \mathrm{m},
\end{aligned}
$$

By using the fuzzy relation $W$, we can reason out fuzzy set $B^{\prime}$ from the input fuzzy set, $A^{\prime}$, which is different from $A$. Each element $b_{j}^{\prime}$ of the reasoned fuzzy set $B^{\prime}$ is given by

$$
\mathrm{b}_{\mathrm{j}}^{\prime}=\max \left[\min \left[\mathrm{a}_{\mathrm{i}}^{\prime}, \mathrm{w}_{\mathrm{i} j}\right]\right], \quad 1 \leqq \mathrm{i} \leqq \mathrm{n}, \quad 1 \leqq \mathrm{j} \leqq \mathrm{m} .
$$

In the experiment, we defined fuzzy sets $A$ and $B$ as vectors $\mathbf{A}$ and $\mathbf{B}$, as shown in Figs. $7(\mathrm{a})$ and $7(\mathrm{~b})$. The given fuzzy rule is represented by the fuzzy relation matrix $\mathbf{W}$, as shown in Fig. 7(c). Figure 7(d) shows the coded pattern of $\mathbf{W}$. The input membership function $\mu\left(A^{\prime}\right)$ is given by the vector $\mathbf{A}^{\prime}$ shown in Fig.

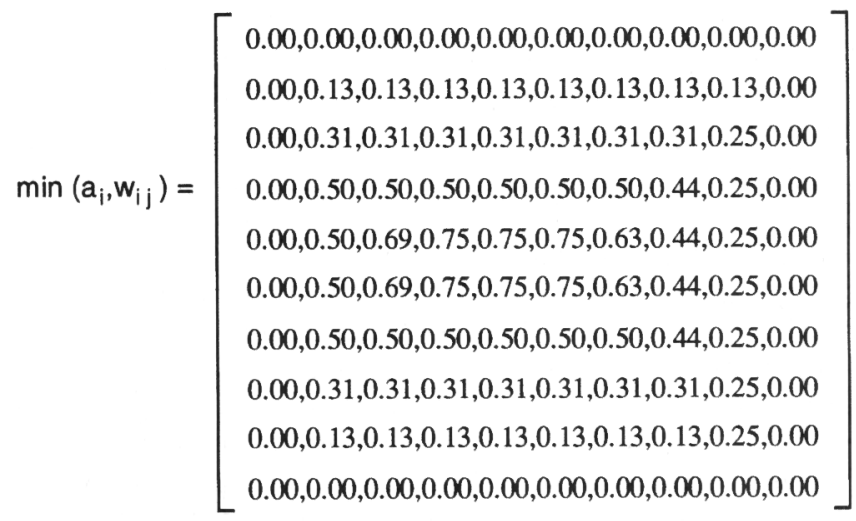

(a)

$\mu\left(B^{\prime}\right): \mathbf{B}^{\prime}=[0.00,0.50,0.69,0.75,0.75,0.75,0.63,0.44,0.25,0.00]$ (b)

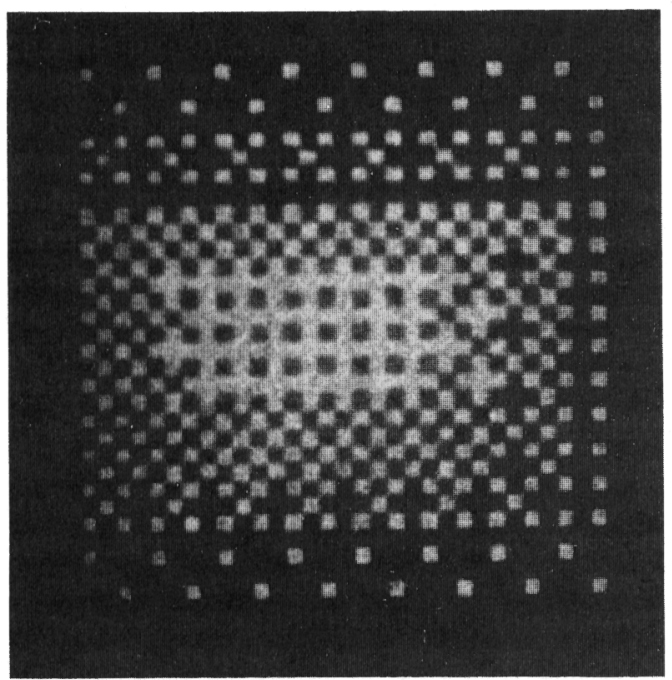

(c)

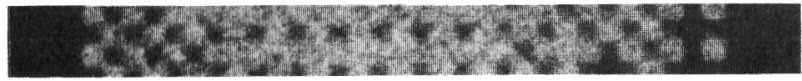

(d)

Fig. 9. Theoretical results of $(a) \min \left\{a_{i}^{\prime}, w_{i j}\right\}$ and $(b) \max \left[\min \left\{a_{i}^{\prime}, w_{i j}\right\}\right]$ and experimental results of $(c) \min \left[a_{i}^{\prime}, w_{i j}\right\}$ and $(d) \max \left[\min \left\{a_{i}, w_{i j} j\right]\right.$.

7(e). Figure 7(f) shows the expansion of the coded pattern of $\mathbf{A}^{\prime}$. Figure 8(a) shows the experimental setup for $\min \left\{a_{\mathrm{i}}^{\prime}, \mathrm{w}_{\mathrm{i} j}\right\}$ in $\mathrm{Eq}$. (7). The coded pattern of $\mathbf{A}^{\prime}$ is illuminated by a plane wave, which is superimposed on the coded pattern of $\mathbf{W}$. By threshol ding the superimposed image with the unit level of intensity, we can get the result of $\min \left\{a_{i}^{\prime}, w_{i j}\right\}$. The theoretical result of $\min \left\{a_{i}^{\prime}, w_{i j}\right\}$ and its experimental result are shown in Figs. 9(a) and 9(c), respectively. Figure $8(b)$ shows the experimental setup for $\max \left[\min \left\{a_{i}^{\prime}, w_{i j}\right]\right]$ in Eq. (7). The result of $\min \left\{a_{i}^{\prime}, w_{i j}\right\}$ is illuminated by $n$ LED's. As a result, the optical distribution is duplicated, shifted, and superimposed on the observed plane. The theoretical and experimental results of $\max \left[\min \left\{a_{i}^{\prime}, w_{i j}\right\}\right]$, i.e., the membership function $\mu\left(B^{\prime}\right)$ reasoned from the input membership function $\mu\left(A^{\prime}\right)$ are shown in Figs. $9(b)$ and $9(d)$, respectively. 


\section{Conclusion}

We have presented a novel technique, the visual-area coding technique (VACT), for the optical implementation of fuzzy logic with the capacity to provide a visualization of the results. This technique is based on the microfont method (MFM) and is considered to be an instance of digitized analog optical computing. Huge amounts of data can be processed in fuzzy logic with the VACT. Moreover, real-time visualization of processed results can be accomplished. Because a lot of pixels must be prepared and processed in the VACT, a highly parallel processing system is required for implementation. The pure optical-parallel-array logic system ${ }^{21}$ is a promising architecture for effective execution of the VACT.

\section{References}

1. J . Tanida and Y. Ichioka, "Optical logic array processor using shadowgrams,"J . Opt. Soc. Am. 73, 800-809 (1983).

2. K.-H. Brenner, A. Huang, and N. Streibl, "Digital optical computing with symbolic substitution," Appl. Opt. 25, 30543060 (1986).

3. K.-S. Huang, B. K. J enkins, and A. A. Sawchuk, "Image algebra representation of parallel optical binary," Appl. Opt. 28, 1263-1278 (1989).

4. M. Fukui and K. Kitayama, "Applications of image-logic algebra: wire routing and numerical data processings,"Appl. Opt. 31, 581-591 (1992).

5. R. G. A. Craig, B. S. Wherrett, A. C. Walker, F. A. P. Tooley, and S. D. Smith, "Optical cellular logic image processor: imple mentation and programming of a single channel digital optical circuit,"Appl. Opt. 30, 2297-2308 (1991).

6. K.-H. Brenner and A. Huang, "Optical implementation of the perfect shuffle interconnection,"Appl. Opt. 27, 135-137 (1988).

7. J . J ahns, "Optical implementation of the Banyan network," Opt. Commun. 76, 321-324 (1990).

8. K.-S. Huang, A. A. Sawchuk, B. K. J enkins, P. Chavel, J .-M. Wang, A. G. Weber, C.-H. Wang, and I. Glaser, "Digital optical cellular image processor (DOCIP): experimental implementation,"Appl. Opt. 32, 166-173 (1993).

9. M. Takahashi, M. Oita, S. Tai, K. Kojima, and K. Kyuma, "A quantized backpropagation learning rule and its application to optical neural networks," Opt. Comput. Process. 1, 175-182 (1991).

10. P. Thiran, V. Peiris, P. Heim, and B. Hochet, "Quantization effects in digitally behaving circuit implementations of Kohonen networks," IEEE Trans. Neural Networks 5, 450-458 (1994).

11. L. Liu, "Optical implementation of parallel fuzzy logic," Opt. Commun. 73, 183-187 (1989).

12. H.-J . Zimmermann, Fuzzy Set Theory and Its Applications (Kluwer-Nijhoff, Boston, Mass., 1985), pp. 121-177.

13. R. Ulichney, Digital Halftoning(MIT, Cambridge, Mass., 1987), pp. 1-14.

14. J. P. Allebach, "Aliasing and quantization in the efficient display of images,"J . Opt. Soc. Am. 69, 869-877 (1979).

15. F. Ono, "Binary rendition of continuous-tone pictures using binary patterns having similar Fourier spectra," Trans. Inst. Electron. Commun. Eng. J pn. Part D J 68D, 686-693 (1985).

16. J . J ahns and M. J . Murdocca, "Cross-over networks and their optical implementation,"Appl. Opt. 27, 3155-3160 (1988).

17. S. Zhang, S. Lin, and C. Chen, "Optical implementation of a fuzzy associative memory," Opt. Commun. 100, 48-52 (1993).

18. Z. Zhu and L. Liu, "Optical cellular continuous Iogic array for gray-scale image processing,"Appl. Opt. 32, 3676-3683 (1993).

19. H. Akirakawa and K. Hirota, "Fuzzy inference engine by address-look-up and paging method," in Proceedings of the International Workshop on Fuzzy System Applications I nternational Fuzzy Systems Association, Amsterdam, North-Holland, 1988), pp. 45-56.

20. Y.-M. Pok, J .-X. Xu, and C. C. Hang, "Visualization of fuzzy control dynamics using vector space," Asia-Pac. Eng. J . A 3, 105-127 (1993).

21. T. Konishi, J. Tanida, and Y. Ichioka, "Pure optical parallel array logic system: an optical parallel computing architecture," Inst. Electron. Info. Commun. Eng. Trans. Electron. E 77-C, 30-34 (1994). 